logues of Lagrange's equations, indispensable matrix of all mechanics.

While admiring these bold works, we fear lest the authors have neglected certain hidden masses, as Helmholtz and Hertz would have said. But although that may happen, there is in these doctrines a curious application of mathematics, which, at least in well-circumscribed cases, has already rendered great services.

I have terminated, messieurs, this summary history of some of the applications of analysis; with the reflections which it has at moments suggested to me.

It is far from being complete; thus I have omitted to speak of the calculus of probabilities, which demands so much subtlety of mind, and of which Pascal refused to explain the niceties to the chevalier de Méré because he was not a geometer.

Its practical utility is of the first rank, its theoretic interest has always been great; it is further augmented to-day, thanks to the importance taken by the researches that Maxwell called statistical and which tend to envisage mechanics under a wholly new light.

I hope, however, to have shown, in this sketch, the origin and the reason of the bonds so profound which unite analysis to geometry and physics, more generally to every science bearing on quantities numerically measurable.

The reciprocal influence of analysis and physical theories has been in this regard particularly instructive.

What does the future reserve?

Problems more difficult, corresponding to an approximation of higher order, will introduce complications which we can only vaguely forecast, in speaking, as I have just done, of functional equations replacing systematically our actual differential equations, or further of integrations of equations infinite in number with an infinity of unknown functions. But even though that happens, mathematical analysis will always remain that language which, according to the mot of Fourier, has no symbols to express confused notions, a language endowed with an admirable power of transformation and capable of condensing in its formulas a number immense of results.

\section{Emile Picard.}

\section{PRESENT PROBLEMS OF METEOROLOGY.*}

Never in the history of the science have so many problems presented themselves for solution as at the present time. Numerous a priori theories require demonstration and, in fact, the whole structure of meteorology, which has been erected on hypotheses, needs to be strengthened or rebuilt by experimental evidence. Until recently the observations have been carried on at the very bottom of the atmosphere and our predecessors have been compared justly to shell-fish groping about the abysses of the ocean floor to which they are confined.

Probably meteorology had its origin in a crude system of weather predictions, based on signs in the heavens, and it did not become a science until the invention of the principal meteorological instruments in the seventeenth century made possible the study of climatology by the collection of exact and comparable observations at many places on the globe. These data, owing to extensive operations of the meteorological services in the different countries, are now tolerably complete, there being comparatively small portions of the land-surface, at least, for which the climatic elements are not fairly well known, the gaps that remain to be filled lying chiefly on the Antarctic continent and in the interior of Africa.

Although it is about fifty years ago since the first observations, made synchronously

* Address at St. Louis to the Section of Cosmical Physies of the International Congress of Arts and Science. 
over a considerable territory, were telegraphed to a central office for the purpose of forecasting the weather, it must be confessed that practically no progress has been realized in this art, for, while much has been done to complete and extend the area under observation by the creation of a finer and larger network of stations, and while the transmission of the observations and the dissemination of the forecasts based on them have been accelerated, the methods employed in formulating the forecasts are essentially those empirical rules which were adopted at the inception of the work. A recent extension of the field of observation over the ocean, by wireless telegraphy, may here be mentioned as offering advantages to certain countries; for example, the reports now being received in England from steamers in mid-Atlantic give information about the approaching weather conditions-subject, of course, to any subsequent changes-long before they reach the western shores of the British Isles. Nevertheless, the data obtained still relate mainly to the lowest strata of the atmosphere and we are ignorant of the conditions that prevail at the height of a mile or two both during storms and in fine weather. Until these are known and their sequence in the upper and lower atmosphere has been established by careful investigation, our weather forecasts based on synoptic observations will continue to be largely empirical. However, it should be remembered that since weather predictions constitute the aspect of meteorology which most appeals to mankind, the incentive to improve them is the most likely to stimulate the investigations needed. Therefore, it is the problems of dynamic meteorology that now press for solution, and to achieve this purpose we must not only look upward, but also elevate ourselves, or our instruments, into the higher regions.
This mode of study belongs entirely to the last half-century, for only within that period has a systematic attempt been made to ascertain the conditions prevailing in the upper air. To the credit of the United States it should be remembered that the first post of observation upon a mountain peak was one established in 1871 upon Mount Washington, in New Hampshire, and this was soon followed by the highest observatory in the world, maintained during fifteen years upon the summit of Pike's Peak in Colorado. The observatory upon the Puy de Dôme in France, opened in 1876, was the first mountain station in Europe to be equipped with self-recording instruments. A large amount of data has been collected at these stations which illustrate chiefly the climatology of the mountainous regions, for what we obtain in this way still pertains to the earth and, as is now admitted, does not represent the conditions prevailing at an equal height in the free air. During the present century, the organized efforts which have been made to explore the ocean of air above us have already resulted in a great increase of knowledge respecting the atmosphere as a whole. This task of ascertaining the conditions of the free air was resumed in 1888, with balloon ascents in Germany, in which special precautions were taken to obtain accurate temperatures, previous observations in balloons leaving much to be desired in this respect. In France, about 1892, it was demonstrated that by means of balloons carrying only self-recording instruments, meteorological information might be acquired at heights far greater than those to which a human being can hope to ascend and live. The use of the so-called 'ballons-sondes,' liberated and abandoned to their fate, with the expectation that when they fall to the ground the records will be recovered, was soon adopted in Germany and has since spread 
all over Europe. It has been introduced into the United States by the writer, who has just despatched the first of these registration-balloons from St. Louis, hoping in this way to obtain the temperatures at heights never before reached above the American continent.

In 1894, at the Blue Hill Observatory, near Boston, kites were first used to lift self-recording instruments and so obtain graphical records of the various meteorological elements in the free air, and this method of observation, which presents the great advantage of securing the data in the different atmospheric strata almost simultaneously and nearly vertically above the station on the ground, has been extensively employed both in this country and abroad. Heights exceeding three miles have been attained and it is possible to ascend a mile or two on almost any day when there is wind. To render the method independent of this factor, the plan of flying kites from a steamship was introduced by the writer three years ago, and this scheme, too, is now being successfully employed in Europe. The exploration of the free air by ballocns and kites, it may be remarked, has given rise to the construction of special types of light and simple self-recording instruments, which are capable of recording automatically the values of temperature, moisture and wind with a precision comparable to the eye-readings of standard instruments by a good observer.

Having described some of the newer methods of meteorological investigation, let us now consider how they may help to solve certain problems in dynamic meteorology. It should be premised that, since the atmosphere is relatively a thin layer with respect to the globe which it covers, no portion of it can be regarded as independent of another and, consequently, a weather-map of the whole globe, day by day, is of prime importance. Were this provided, the atmospheric changes occurring simultaneously in both hemispheres could be watched and their relation to what have been called 'the great centers of action,' investigated. Thanks to the increasing area covered by reports from the various weather services, the unmapped surface of the globe is being diminished, so that a complete picture of the state of the atmosphere each day over the land is gradually coming into view.

The mathematical application of the theory of a rotating sphere, surrounded by a heated atmosphere, to explain the circulation of the atmosphere as we find it, has not been satisfactory, owing to our lack of knowledge of the conditions of the upper air, as well as our ignorance concerning the physical properties of the atmosphere itself. To acquire the latter knowledge, research laboratories must be established at selected points, at both high and low levels, and as subjects of study there may be mentioned the determination of the amount of heat received from the sun and its secular variation, if any, the radiating and absorbing power of the air, the relation of pressure, density and temperature, the chemical composition of the air, its ionization and radioactivity, and other investigations which have been proposed by Professors Abbe and McAdie in their pleas for the creation of such aerophysical laboratories. The observatory now under construction by the United States Weather Bureau on a mountain in Virginia will, it is hoped, enable some of these problems to receive the attention which they deserve.

The average circulation of the lower atmosphere is now well known, by reason of the monumental work of Lieutenant Maury on the winds over the oceans, and from the mass of data since collected over oceans and continents through the meteorological organizations of the various coun- 
tries. While, naturally, much less is known regarding the circulation of the upper air, a great deal has been ascertained from the observations of clouds that were instituted a few years ago in various parts of the world by an international committee. In order to insure that the same cloud should everywhere be called by the same name, it was necessary to instruct the observers by publishing a cloud-atlas, containing pictures and descriptions of the typical forms of clouds which experience has shown to be identical all over the globe. Then during one year which had been agreed upon, measurements of the direction of drift and the apparent velocity of the several cloud-types were made at many stations, and measurements, by trigonometrical or other methods, of the height of these clouds above a few selected stations enabled the true velocity of the air-currents to be determined up to the altitude at which the cirrus clouds float. Thus an actual survey of the direction and speed of the atmospheric circulation at different levels was effected, and a recent discussion of the results by Professor Hildebrandsson shows that the theories which have been held heretofore are untenable. Professor Hildebrandsson's conclusions in brief are that there is no exchange of air between poles and equator, the circulation over the oceans, at least, resolving itself into four great whirls, the air which rises above the tropics flowing over the trades and descending probably in the extra-tropical regions, while around each pole is an independent cyclonic circulation. Although this general circulation of the atmosphere appears to be indicated, many details require to be investigated. In particular, the movements of the great masses of air overlying the trade winds and doldrums, which is a region nearly barren of clouds, are still unknown and the determination of these movements, as well as the temperature and humidity of the different strata, by means of kites flown from steamships, was suggested by the writer, since it would be possible in this way to penetrate even the masses of quiescent air which probably separate the trade winds from the superposed antitrades. This suggestion has already been put in practise on the yacht of the Prince of Monaco in the neighborhood of the Azores, but a more extensive campaign is necessary, which the writer himself hopes to undertake, if the funds necessary to charter and equip a steamer can be procured.

Here it will be encouraging to state some results of the efforts to ascertain the vertical thermal and hygrometric gradients in the atmospheric ocean, and to show what may be accomplished in the future. Observations on mountains, as we have seen, can not be expected to give the conditions which exist at the corresponding heights in the free air and hence the necessity of sending observers or self-recording instruments into this medium through the agency of balloons and kites. By the aid of an international committee formed eight years ago under the direction of Professor Hergesell at Strassburg, much has been accomplished in Europe in this way, and something in this country through kite-flights. At the present time such atmospheric soundings are made once a month in most European countries, and at Blue Hill in the United States, with the result that a knowledge is being acquired of the vertical gradients of the meteorological elements which entirely contradicts previous conceptions. For example, it was formerly supposed that the temperature diminished with increasing altitude more and more slowly and that at a height of about ten miles it remained invariable during winter and summer and above pole and equator. But the recent investigations of my colleagues in France and Germany show that the temperature 
decreases faster and faster as one rises in the air and that not only is there a large seasonal variation at the greatest heights attained, but that non-periodic changes occur from day to day, as they do at the earth's surface. Still more remarkable is the indication of a warm current at a height of about seven miles, while the stratification of the atmosphere as regards temperature, moisture and wind has been shown by the kite-flights at Blue Hill to be a normal condition, and not merely confined to the high atmosphere as was formerly supposed. Daily soundings of the atmosphere to the height of a mile or two are now being made with kites and captive balloons at the meteorological institutes of Berlin, Hamburg and St. Petersburg, and are furnishing valuable data concerning the changes in the meteorological elements which occur simultaneously or successively in the overlying strata.

Of the various unsolved questions relating to this subject perhaps the most important is whether the core of the cyclone possesses the excess of temperature over the surrounding body of air which the convectional theory of its origin requires. We need to know also the height to which the cyclone extends, the circulation around it at various levels, and further to generalize the theory of an accompanying cold-center cyclone in the upper air, deduced by Mr. Clayton from the Blue Hill observations. Other vexed questions can be elucidated by similar researches, such as the conditions favorable for precipitation and the action of dust-nuclei in producing it, the source of our American cold-waves, the exact relations of thunderstorms and tornadoes to centers of pressure and temperature and, finally, the causes which, in the upper air, influence the trajectories and velocities of the cyclones and anti-cyclones that give us our broader weather features. When these correlations are determined from the in- vestigations of the free air now in progress, and we possess a sufficient number of aerial stations to make it possible to chart a daily map of the upper air, then we may expect an improvement in the weather forecasts. 'The prediction of fog over the ocean on, and adjacent to, our coasts is of great practical importance to shipping, especially off the banks of Newfoundland, and the writer believes that meteorological kites flown from a steamer in these regions would reveal the unknown relations of temperature, humidity and wind in and above the fog-bank which might lead to the prediction of the conditions favorable to its formation.

We now pass to another branch of meteorological research, namely, the cosmical relations. It is incontestable that the sun, the source of all terrestrial energy, has great influence upon the magnetic conditions of the earth, but a consideration of the relation of terrestrial magnetism and meteorology will be left to my colleague, Dr. Bauer. The cause of atmospheric electricity has always been an enigma to meteorologists, but the discovery of 'ions,' or 'electrons,' as carriers of electricity, has thrown some light on this question. It' is of importance in geophysics to know how the capacity of the air for positive and negative electrons varies with altitude, and also the periodic and nonperiodic variation of the potential at the earth's surface.

Attempts to regard all atmospheric phenomena as periodic and due to the influence of the sun or moon have long occupied the attention of eminent investigators, but it must be admitted that the effects of neither the periods of solar nor those of lunar rotation upon the earth's meteorology can be claimed to have been proved, although a correspondence has been found by the distinguished speaker who preceded me in regard to the frequencies of auroras and 
thunderstorms and the position of the moon in declination. To Professor Arrhenius is also due the remarkable generalization that the pressure of light emanating from the sun causes alike the streaming away from it of comets' tails, the zodiacal light and the aurora borealis. The relation of sunspot frequency; which has a periodicity of about eleven years, to atmospheric changes on the earth, especially as manifested by barometric pressure, rainfall and temperature in India, has been investigated, and the coincidences, even if nothing more, which have been shown to exist by Sir Norman Lockyer and his son are suggestive. It may be pointed out that the same action of the sun might cause simultaneously increased rainfall in India and a deficiency of rainfall in England, because rising currents in one region are necessarily accompanied by descending currents elsewhere, and, therefore, no objection can be offered to a theory of cosmical influence which produces different weather conditions in different parts of the globe.

Since the sun is the source of our energy, the discovery of any variation in the heat emitted is of the deepest interest and the important investigations of Professor Langley are now to be supplemented by the broader work of a committee appointed by the National Academy, of Sciences and also by an international committee, with the general object of combining and discussing meteorological observations from the point of view of their relation to solar phenomena. It does not seem improbable, therefore, that eventually we may have seasonal predictions of weather, possessing at least the success of those now made daily, and that possibly forecasts of the weather will be hazarded several years in advance. The value of such forecasts, as affecting the crops alone, would be of inestimable benefit to mankind, and predictions already made in India for the ensuing season, while not entirely successful, have still proved advantageous. A number of short cycles in the weather have been detected, including a seven-day period in the temperature. which Mr. Clayton found could be used for forecasting were it not for an unexplained reversal in the phase of the temperature oscillation.

The interesting question of the value of meteorological observations may appropriately conclude this address. Professor Schuster, the English physicist, has recently denounced the practice of accumulating these observations with no specific purpose. To an extent this criticism is valid in all the sciences, since those observations are most useful when made by or for the person who is to utilize them, but although modern meteorology demands special series of observations to solve such problems as the temperature in cyclones and anticyclones, it is sometimes true that long series of observations made with one object in view may subsequently become valuable for quite another purpose. For the study of climate and its possible change, long-continued observations in each country are a necessity, though these might properly be confined to selected stations from whose normals the values for other stations may be computed. Professor Schuster's wish to limit the number of observations implies that the existing series have been inadequately discussed, for the reason that it is easier to find observers than competent investigators. For this unfortunate condition the weather services of most countries are chiefly to blame, because, being burdened with the routine work of collecting climatological and synoptic data and formulating and promulgating weather forecasts, which is the public estimate of their entire duty, most government meteorological organizations concentrate their energies and expenditures on these functions and par- 
tially or completely neglect the researches by which alone our knowledge of the mechanies of the atmosphere can be increased. In this criticism must be included the United States Weather Bureau (exception being made in favor of Professor Bigelow's discussions), and the similar bureaus of such equally enlightened countries as France and England. However, in the latter country an attempt is now being made to create an Imperial meteorological institute which could undertake the discussion of the great mass of data accumulated in Great Britain and her colonies, especially the relations of solar phenomena to meteorology and magnetism, and it is argued that this would contribute towards the formation of a body of scientific investigators adequate to the needs of the British Empire and be of the highest educational and scientific worth. In the United States, meteorological research has always been fostered by individuals, of whom the names of Franklin, Redfield, Espy, Coffin, Maury, Loomis and Ferrel are brilliant examples. To-day my cclleague, M. Teisserenc de Bort in France, and we ourselves at Blue Hill, are endeavoring, unassisted, to solve problems in dynamic meteorology, which ought to be undertaken by the national services of our respective countries. It behooves then those who are desirous of advancing the status of meteorology to strive to convince the public that the function of a government bureau is not merely to collect meteorological data and to make inductive weather predictions based on remembrance of the sequence in similar conditions, but that the science of meteorology requires laborious researches by competent men and the generous expenditure of money before practical benefit can result from improved weather forecasts. If some of my hearers are converted to such an opinion, this address will have served a useful purpose.

A. Lawrence Rotch.

Blue Hill Meteorological Observatory.

\section{SCIENTIFIC BOOKS.}

The Lymphatics. By PoIrier, Cunéo and

Delamere. Translated and edited by Cecil H. Leaf. Chicago, W. T. Keener \& Co. 1904.

The chapters on the 'Lymphatic System' in Poirier's 'Anatomy' have been translated into English and presented in book form by C. H. Leaf. The first half of the book is on the 'General Anatomy of the Lymphatics,' and is by Delamere, while the second half is by Poirier and Cunéo and treats of the 'Special Study of the Lymphatics in Different Parts of the Body.' The translation is thoroughly well done.

The increasing interest in the lymphatic system makes the appearance of such a book especially welcome. It is the most complete and comprehensive monograph on the lymphatic system that we have and will fill a longfelt need. It contains some original work, especially the last half. The two parts are distinct and will be taken up separately.

The plan of the first part is excellent, the subject being treated systematically under four heads, the lymph, the leucocytes, the lymphatic vessels and the glands. Each subject is treated first practically, by giving our definite knowledge about it, and then theoretically. The practical part is excellent, clear, comprehensive, definite and not diffuse. It brings together facts for which one would otherwise have to hunt through many books. The treatment of the theoretical part, on the other hand, is weak. For example, under the lymph, its properties, physical and chemical, are unusually well given; but in the treatment of the theories of the formation of lymph it is not clearly brought out that there are two opposing theories; one that physical forces are sufficient to account for the formation of lymph, and the other that it is a secretion of endothelial cells. It is not shown that the physical theory, which has grown out of the 